\title{
ESTUDO COMPARATIVO DA PRESSÃO ARTERIAL E DA PREVALENCIA DE HIPERTENSÃO ARTERIAL EM DUAS COORTES SUCESSIVAS (1975-1976) DE ESTUDANTES DE 16 A 25 ANOS, BOTUCATU, SP, BRASIL *
}

\author{
Dináh Borges de Almeida** \\ Francisco Habermann** \\ Vitor Augusto Soares** \\ Renato Costa Monteiro Filho*** \\ Eduardo Simōes Ferreira*** \\ Omar Abujamra Junior *** \\ Sheila Zambello de Pinho $* * * *$ \\ Cecilia Magaldi $* * * * *$
}

AlmeidA, D. B. de et al. Estudo comparativo da pressão arterial e da prevalência de hipertensão arterial em duas coortes sucessivas (1975-1976) de estudantes de 16 a 25 anos, Botucatu, SP, Brasil. Rev. Saúde públ., S. Paulo, 12:497-505, 1978.

RESUMO: Com o objetivo de determinar os niveis de pressão arterial $e$ a prevalência de hipertensão arterial em uma população jovem, foram realizados pesquisas em dois anos sucessivos em 1.288 e 736 estudantes de Botucatu, SP (Brasil) tendo sido comparados os resultados obtidos. As médias das pressóes sistólicas da população estudada e dos dois grupos etários desta população (16 a 20 anos e 21 a 25 anos') foram identicas em ambos os anos, tendo as médias das pressões diastólicas diferido de no máximo $2 \mathrm{mmHg}$; as médias, tanto sistólicas quanto diastólicas, dos dois sexos e da parcela branca da população estudada quanto à idade e sexo também diferiram de no máximo $2 \mathrm{mmHg}$. As médias da população estudada e sua parcela branca, em ambos os anos, foram superiores no sexo masculino e no grupo etário de 21 a 25 anos. $N a$ população negra e amarela houve disparidade de resultados entre 1975 e 1976 , indicando influência da exiguidade do tamanho dos contigentes negro $e$ amarelo desta população. A prevalência de hipertensão arterial (pressão sistólica igual ou maior que $140 \mathrm{mmHg}$ e diastólica igual ou maior que $90 \mathrm{mmHg}$ ) foi de $5,04 \%$ em 1975 e $6,22 \%$ em 1976 , tendo sido em ambos os anos maior no sexo masculino do que no feminino e no grupo de 21 a 25 do que no de 16 a 20 anos.

Unitermos: Hipertensão. Pressão arterial. Estudantes, Botucatu, SP, Brasil.

* Trabalho realizado na Faculdade de Medicina de Botucatu da Universidade Estadual Paulista (UNESP), Botucatu, SP com auxillio financeiro da Fundação W. K. Kellogg.

* Do Departamento de Clínica Médica - Faculdade de Medicina de Botucatu da UNESP. Botucatu, SP - Brasil.

*** Acadêmico de Medicina da Faculdade de Medicina de Botucatu da UNESP, Botucatu, SP - Brasil.

*** Do Departamento de Matemática do Instituto Básico de Biologia Médica e Agrícola e Centro de Processamento de Dados do Campus de Botucatu, da UNESP, São Paulo, SP - Brasil.

***** Do Departamento de Medicina Legal e Medicina em Saúde Pública da Faculdade de Medicina de Botucatu, da UNESP. Botucatu, SP - Brasil. 
ALMEIDA, D. B. de et al. Estudo comparativo da pressão arterial e da prevalência de hipertensão arterial em duas coortes sucessivas (1975-1976) de estudantes de 16 a 25 anos, Botucatu, SP, Brasil. Rev. Saúde públ., S. Paulo, 12:497-505, 1978.

\section{INTRODU ÇÃO}

São escassos no Brasil os estudos sistemáticos sobre os niveis de pressão arterial sistêmica (PA) da população brasileira e de prevalência de hipertensão nesta população $2,3,4,6,7,9,11,12,16$, a despeito de ser a hipertensão arterial um sério problema de Saúde Pública ${ }^{15}$. Com exceção dos trabalhos de Gonzalez ${ }^{11}$ e Colli e col. ${ }^{9}$, que realizaram investigações com o objetivo de determinar os niveis de pressão arterial de escolares de duas grandes cidades brasileiras e o de Agne e col.2, os poucos dados conhecidos sobre níveis de pressão arterial de componentes da população brasileira são derivados de estudos da prevalência de cardiopatias 12,17. Por outro lado, alguns informes sobre a prevalência da hipertensão têm derivado de trabalhos que estudam a morbidade geral 1,19. Alguns estudo foram realizados na população em demanda a hospitais 8,14 .

Tendo em vista que Cortez Jr. ${ }^{10}$ demonstrou ser a hipertensão a $5^{\text {a }}$ causa básica de óbito no Municipio de Botucatu e a primeira segundo causas múltiplas, resolveu-se empreender pesquisa em estudantes de $2^{\circ}$ ciclo, com os seguintes objetivos: determinar os níveis de PA em jovens de zona urbana, de tal forma a contribuir para um delineamento dos perfis de PA dos vários componentes da população brasileira; determinar a prevalência da hipertensão entre jovens e encaminhar os hipertensos descobertos para investigação clínica e orientação terapêutica. Foram realizadas determinações em dois anos sucessivos, 1975 e 1976 . Os resultados relativos a 1975 foram anteriormente publicados ${ }^{3}$. 0 objetivo do presente trabalho é divulgar os referentes à 1976 e analisar comparativamente os obtidos em ambos os anos.

\section{MATERIAL E MÉTODOS}

Foram entrevistados e foi medida a PA dos alunos de ambos os sexos do $2^{\circ}$ e $3^{\circ}$ anos do $2^{\circ}$ ciclo de todos os seis estabelecimentos de ensino deste nível e $1^{\circ}$ ano das duas Faculdades existentes em Botucatu. Não foram examinados todos os alunos matriculados na época da pesquisa por não estarem alguns comparecendo às aulas (evasão escolar). Dentre os alunos examinados foram incluídos aqueles cujas idades situavam-se entre 16 a 25 anos inclusive. Os alunos examinados em 1975 e reexaminados em 1976 foram incluidos apenas na casuística de 1975. A Tabela 1 apresenta a população de referência, a examinada e a incluída no estudo em 1975 e 1976, e na Tabela 2 encontra-se a distribuição segundo a idade, sexo, cor e peso. A metodologia empregada na pesquisa de 1976 foi idêntica a de 1975, publicada anteriormente 3 .

T A B E L A 1

Populações de referência, populações examinadas e populações incluídas no estu to em 1975 e 1976

\begin{tabular}{|c|c|c|c|c|}
\hline População estudada & 1975 & $(\%)$ & 1976 & $(\%)$ \\
\hline Total alunos & 1566 & - & 971 & - \\
\hline Examinados & 1397 & $(89,2)$ & 814 & $(83,8)$ \\
\hline Incluidos & 1288 & - & 736 & - \\
\hline Sexo masculino & 599 & $(46,5)$ & 358 & $(48,6)$ \\
\hline Sexo feminino & 689 & $(53,4)$ & 378 & $(51,3)$ \\
\hline Idade 16 a 20 & 962 & $(74,6)$ & 582 & $(79,0)$ \\
\hline Idade 21 a 25 & 326 & $(25,3)$ & 154 & $(20,9)$ \\
\hline
\end{tabular}




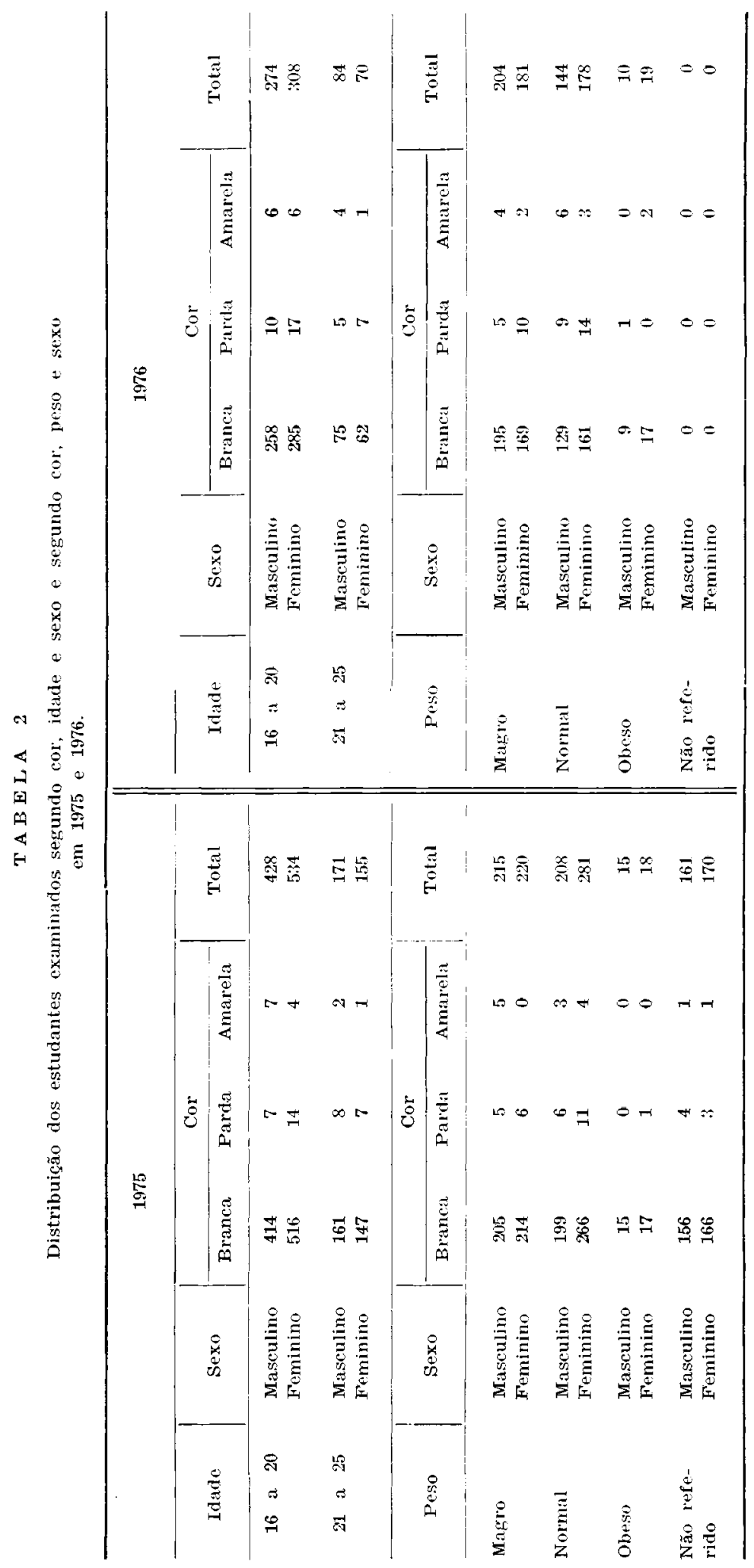


ALMEIDA, D. B. de et al. Estudo comparativo da pressão arterial e da prevalência de hipertensão arterial em duas coortes sucessivas (1975-1976) de estudantes de 16 a 25 anos, Botucatu, SP, Brasil. Rev. Saúde públ., S. Paulo, 12:497-505, 1978.

$\mathrm{Na}$ Tabela 3 está especificada a classificação adotada para a PA.

\section{RESULTADOS}

Na Tabela 4 são apresentadas as médias das pressōes sistólicas e diastólicas e respectivos desvios padrões obtidos nos dois anos para a população total investigada, para cada sexo e para as duas faixas etárias estudadas. Observa-se que as médias das pressões sistólicas foram idênticas em ambos os anos na população estudada e nas duas faixas etárias, tendo diferido de apenas $1 \mathrm{mmHg}$ entre os dois anos em ambos os sexos. Já a média das pressões diastólicas diferiu de um ano para outro em todos os grupos, embora esta variação tenha sido no máximo de $2 \mathrm{mmHg}$.

Tanto as médias das pressóes sistólicas quanto as das diastólicas em ambos os anos foram maiores no sexo masculino do que no feminino e no grupo etário de 21 a 25 em relação ao de 16 a 20 anos. As dife- renças das médias das pressões sistólicas foram mais pronunciadas do que as das pressões diastólicas.

$\mathrm{Na}$ Tabela 5 estão distribuídas as médias da PA de acordo com idade, sexo e cor.

Observa-se que, na cor branca, os resultados obtidos em 1975 e 1976 foram similares, as médias da PA diferiram no máximo de $2 \mathrm{mmHg}$ em ambos os sexos e faixas etárias e que as tendências observadas, na população geral, de médias superiores no sexo masculino e faixa etária de 21 a 25 anos, se mantiveram.

Para os de cor parda e amarela houve disparidade dos resultados obtidos nos dois anos; por outro lado, não houve um comportamento uniforme das médias quanto ao sexo e idade. Assim, em relação aos pardos, segundo o sexo, enquanto nos dois anos as médias da PA foram superiores no sexo masculino na faixa etária de 21 a 25 anos, no grupo de 16 a 20 em 1975, as médias do sexo feminino foram superiores às do sexo masculino. Nesta mesma

T A B E L A 3

Classificação da Pressão Arterial adotada no estudo dos niveis de pressão arterial de estudantes de 16 a 25 anos.

\begin{tabular}{|c|c|c|c|}
\hline Classificação Pressórica & PAS & $(\mathrm{mmHg})$ & PAD (mmHg) \\
\hline Normotenso & & $<135$ & $<85$ \\
\hline Limitrofe Sistólico & 135 & $-\longrightarrow 140$ & $<85$ \\
\hline Limítrofe Diastólico & & $<135$ & $851-90$ \\
\hline $\begin{aligned} \text { Limítrofe } & \text { Sistólico e } \\
& \text { Diastólico }\end{aligned}$ & 135 & $\longrightarrow 140$ & $851 \longrightarrow 90$ \\
\hline Hipertenso Sistólico & & $>140$ & $<85$ \\
\hline Hipertenso Diastólico & & $<135$ & $>90$ \\
\hline $\begin{aligned} \text { Hipertenso } & \text { Sistólico e } \\
& \text { Diastólico }\end{aligned}$ & & $>140$ & $>90$ \\
\hline $\begin{array}{l}\text { Hipertenso Sistólico e } \\
\text { Limítrofe Diastólico }\end{array}$ & & $>140$ & $85 \longmapsto-190$ \\
\hline $\begin{array}{c}\text { Hipertenso Diastólico e } \\
\text { Limítrofe Sistólico }\end{array}$ & 135 & $\longrightarrow 140$ & $>90$ \\
\hline
\end{tabular}

PAS = pressão arterial sistólica

$\mathrm{PAD}=$ pressão arterial diastólica 
ALMEIDA, D. B. de et al. Estudo comparativo da pressão arterial e da prevalência de hipertensão arterial em duas coortes sucessivas (1975-1976) de estudantes de 16 a 25 aros, Botucatu, SP, Brasil. Rev. Saúde públ., S. Paulo, 12:497-505, 1978.

T A B E A 4

Médias da pressão arterial e desvios pađrões por sexo e idade e média geral da pressão arterial.

\begin{tabular}{|c|c|c|c|c|c|}
\hline Sexo e idade & Ano & PAS & $(\mathrm{mmHg})$ & PAD & $(\mathrm{mmHg})$ \\
\hline Masculino & $\begin{array}{l}1975 \\
1976\end{array}$ & $\begin{array}{l}120 \\
119\end{array}$ & $\begin{array}{l} \pm \quad 12 \\
\pm \quad 12\end{array}$ & $\begin{array}{l}74 \\
75\end{array}$ & $\begin{array}{l} \pm 9 \\
\pm 9\end{array}$ \\
\hline Feminino & $\begin{array}{l}1975 \\
1976\end{array}$ & $\begin{array}{l}114 \\
115\end{array}$ & $\begin{array}{ll} \pm & 11 \\
\pm & 12\end{array}$ & $\begin{array}{l}72 \\
74\end{array}$ & $\begin{array}{l} \pm 9 \\
\pm 9\end{array}$ \\
\hline $16 \longrightarrow 20 \mathrm{a}$ & $\begin{array}{l}1975 \\
1976\end{array}$ & $\begin{array}{l}116 \\
116\end{array}$ & $\begin{array}{l} \pm 11 \\
\pm 12\end{array}$ & $\begin{array}{l}73 \\
74\end{array}$ & $\begin{array}{l} \pm 9 \\
\pm 9\end{array}$ \\
\hline $21 \longrightarrow 25 \mathrm{a}$ & $\begin{array}{l}1975 \\
1976\end{array}$ & $\begin{array}{l}119 \\
119\end{array}$ & $\begin{array}{l} \pm 13 \\
\pm 12\end{array}$ & $\begin{array}{l}74 \\
76\end{array}$ & $\begin{array}{l} \pm 9 \\
\pm 9\end{array}$ \\
\hline Geral & $\begin{array}{l}1975 \\
1976\end{array}$ & $\begin{array}{l}117 \\
117\end{array}$ & $\begin{array}{l} \pm 12 \\
\pm 12\end{array}$ & $\begin{array}{l}73 \\
74\end{array}$ & $\begin{array}{l} \pm 9 \\
\pm 9\end{array}$ \\
\hline
\end{tabular}

PAS = média da pressão arterial sistólica \pm desvio padrão

$\mathrm{PAD}=$ média da pressão arterial diastólica \pm desvio pađrão

cor, no sexo masculino em ambos os anos, e no feminino em 1976, as médias da faixa etária de 21 a 25 anos foram superiores às do grupo de 16 a 20 anos, enquanto que no sexo feminino em 1975 as médias foram superiores na faixa etária de 16 a 20 , em relação às da faixa etária de 21 a 25 anos. $\mathrm{Na}$ cor amarela não foi possivel identificar nenhuma tendência das médias quanto ao sexo e idade.

$\mathrm{Na}$ Tabela 6 estão distribuidas as médias da PA segundo sexo, cor e peso. Observa-se em ambos os sexos, na cor branca e parda, uma tendência ao aumento das médias com o aumento de peso, tendência esta que se manifesta mais intensamente entre os de peso normal e os obesos.

$\mathrm{Na}$ Fig. 1 estão representadas as prevalências dos vários tipos de comportamento da PA, segundo a classificação adotada. Observa-se que a prevalência tanto de limitrofes quanto de hipertensos foi maior em 1976 do que em 1975. Por outro lado, dentre as alterações da PA houve predomínio de limítrofes diastólicos e a seguir de sistólicos, nos dois anos.

$\mathrm{Na}$ Fig. 2 estão representadas as prevalências da hipertensão segundo idade e sexo. Em ambos os anos, a prevalência de hipertensão foi maior na faixa etária de 21 a 25 anos do que na de 16 a 20 e no sexo masculino do que no feminino.

\section{COMENTARIOS}

A realização da pesquisa de 1976 permitiu avaliar a consistência dos resultados obtidos em 1975 bem como das conclusões a que o referido trabalho possibilitou chegar".

A análise comparativa de ambas as pesquisas mostrou que as conclusões referentes à população estudada e à sua parcela de cor branca, quanto à média da PA (geral), aos níveis médios por sexo e idade e a tendência dos níveis serem mais elevados no sexo masculino do que no feminino e na faixa etária de 21 a 25 anos do que na de 16 a 20 são válidas para os dois trabalhos. A quase identidade de todos estes valores, entre os dois anos, demonstra que a metodologia estabelecida foi respeitada durante as duas pesquisas; ressalte-se que deve ter contribuido para isto o fato de ambas terem sido levadas a efeito pelo mesmo grupo de entrevistadores.

A similaridade dos resultados obtidos em 
TA B E I A 5

Distribuição de médias da pressão arterial e desvios padrões segundo a idade, sexo, cor e ano.

\begin{tabular}{|c|c|c|c|c|c|c|c|c|c|c|}
\hline \multirow{2}{*}{ Idade } & \multirow{2}{*}{ Ano } & \multicolumn{4}{|c|}{ Sexo masculino } & \multicolumn{5}{|c|}{ Sexo feminino } \\
\hline & & PAS $(\mathrm{mmHg})$ & PAD & $(\mathrm{mmHg})$ & $N$ & PAS & $(\mathrm{mmHg})$ & PAD & $(\mathrm{mmHg})$ & $\mathrm{N}$ \\
\hline \multirow{3}{*}{$16-20$} & \multicolumn{10}{|c|}{ Cor branca } \\
\hline & 1975 & $119 \pm 12$ & 73 & \pm 9 & 414 & 114 & \pm 10 & 72 & \pm 8 & 516 \\
\hline & 1976 & $118 \pm 12$ & 74 & \pm 8 & 258 & 115 & \pm 12 & & \pm 9 & 285 \\
\hline \multirow{3}{*}{$21-25$} & 1975 & $121 \pm 13$ & 75 & \pm 8 & 161 & 115 & \pm 11 & & \pm 9 & 147 \\
\hline & $19 ; 6$ & $121 \pm 11$ & 76 & \pm 8 & 75 & 117 & \pm 13 & & \pm 8 & 62 \\
\hline & \multicolumn{10}{|c|}{ Cor parda } \\
\hline \multirow{2}{*}{$16-20$} & 1975 & $115 \pm 5$ & 72 & \pm 11 & 7 & 118 & \pm 14 & & \pm 9 & 14 \\
\hline & 1976 & $115 \pm 18$ & 75 & \pm 9 & 10 & 113 & \pm 11 & & \pm 6 & 17 \\
\hline \multirow{3}{*}{$21-25$} & 1975 & $126 \pm 14$ & 86 & \pm 8 & 8 & 112 & \pm 5 & & \pm 3 & 7 \\
\hline & 1976 & $122 \pm 7$ & 81 & \pm 7 & 5 & 115 & \pm 8 & & \pm 6 & 7 \\
\hline & \multicolumn{10}{|c|}{ Cor amarela } \\
\hline \multirow{2}{*}{$16-20$} & 1975 & $119 \pm 16$ & 74 & \pm 7 & 7 & 117 & \pm 4 & & \pm 4 & 4 \\
\hline & 1976 & $119 \pm 14$ & & \pm 14 & 6 & 122 & \pm 12 & & \pm 12 & 6 \\
\hline \multirow{2}{*}{$21-25$} & 1975 & $117 \pm 18$ & & \pm 7 & 2 & 110 & & 84 & & 1 \\
\hline & 1976 & $123 \pm 13$ & 85 & \pm 13 & 4 & & - & & - & - \\
\hline
\end{tabular}

PAS = média da pressão arterial sistólica \pm desvio padrão

$\mathrm{PAD}=$ média da pressão arterial diastólica \pm desvio padrão

$\mathrm{N}=$ frequência absoluta

TA B ELA 6

Distribuição de médias da pressão arterial e desvios padrões segundo sexo, cor, peso e ano.

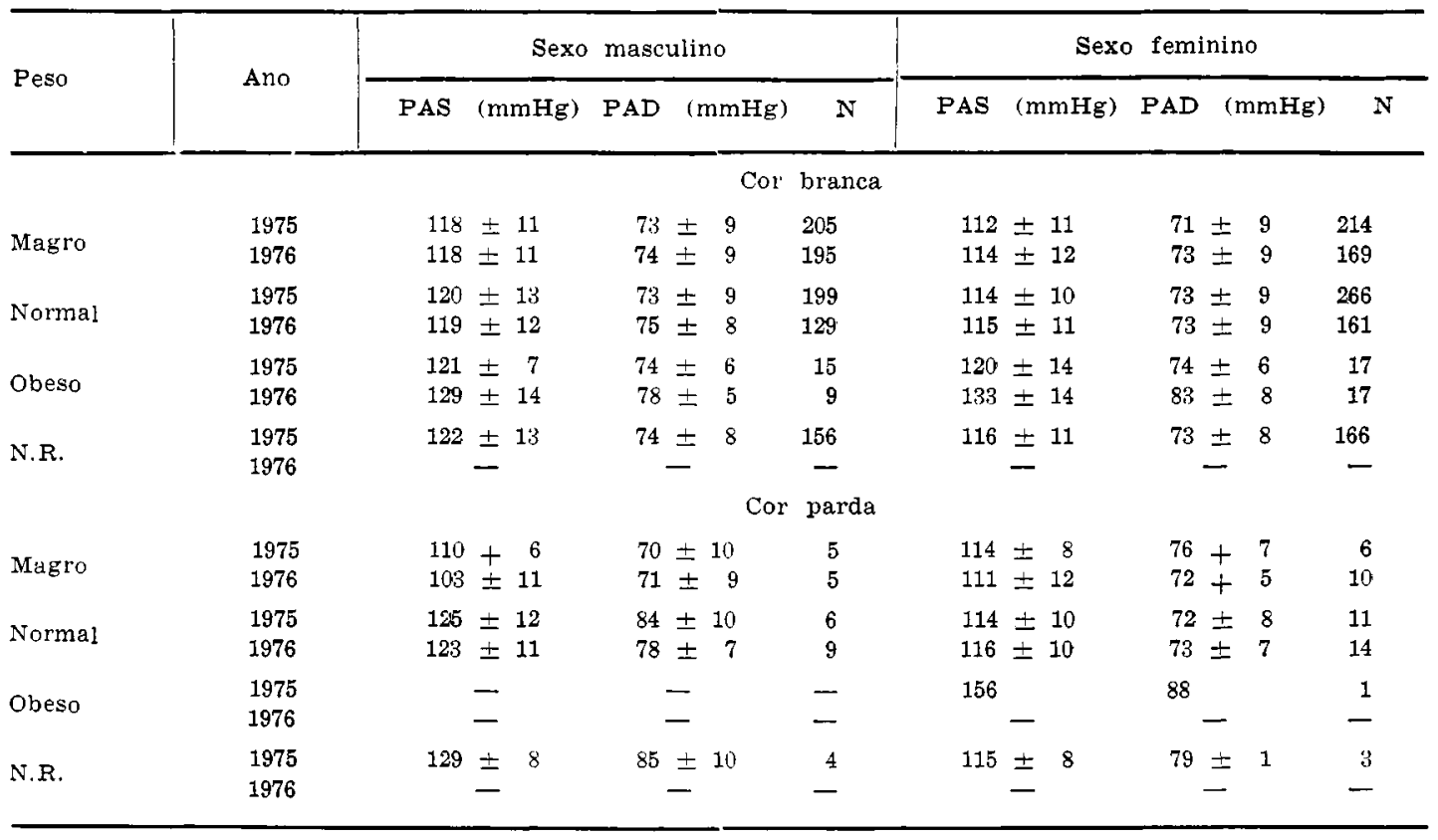

PAS = média da pressão arterial sistólica \pm desvio padrão

$\mathrm{PAD}=$ média da pressão arterial diastólica \pm desvio padrão

$N=$ frequência absoluta

N.R. = peso não referido 
ALMEIDA, D. B. de et al. Estudo comparativo da pressão arterial e da prevalência de hipertensão arterial em duas coortes sucessivas (1975-1976) de estudantes de 16 a 25 anos, Botucatu, SP, Brasil. Rev. Saúde públ., S. Paulo, 12:497-505, 1978.

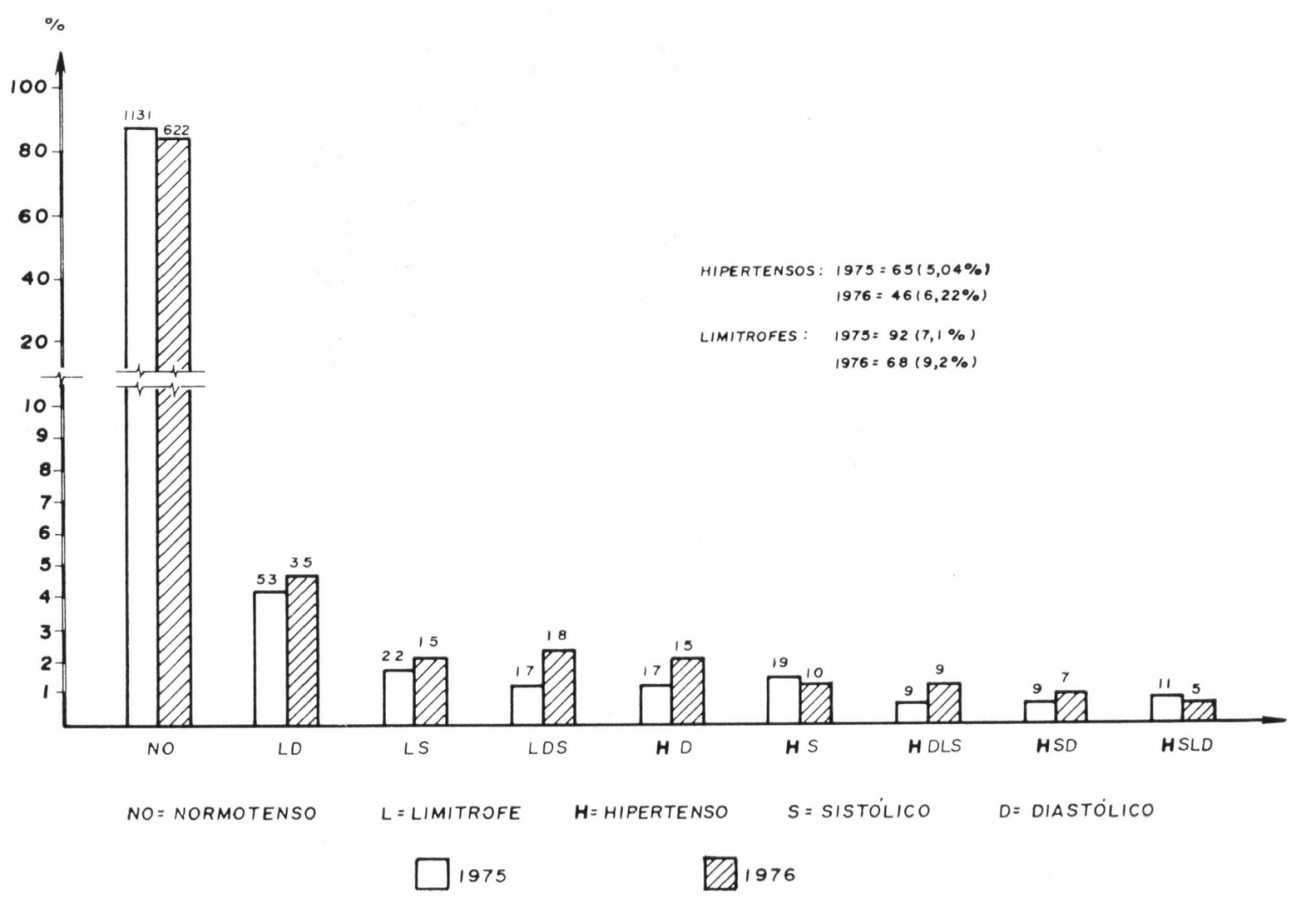

Fig. 1 - Classificação da pressão arterial dos estudantes examinados em pesquisas realizadas na cidade de Botucatu, em 1975 e 1976.
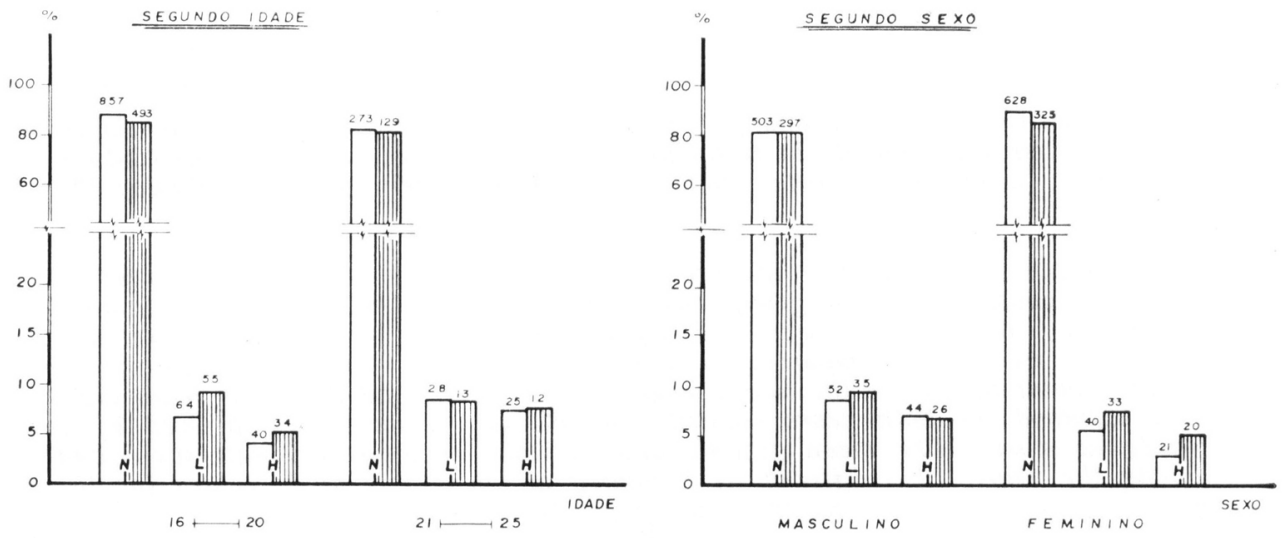

N NORMOTENSOS

L LIMITROFES

H HIPERTENSOS

Fig. 2 - Classificação da pressão arterial segundo idade e sexo dos estuatantes examinados em pesquisas realizadas na cidade de Botucatu, em 1975 e 1976. 
ALMEIDA, D. B. de et al. Estudo comparativo da pressão arterial e da prevalência de hipertensão arterial em duas coortes sucessivas (1975-1976) de estudantes de 16 a 25 anos, Botucatu, SP. Brasil. Rev. Saúde públ., S. Paulo, 12:497-505, 1978.

1975 e 1976 para a população estudada e para sua parcela de cor branca, conforme expressa a Tabela 5 , permite afirmar que os resultados obtidos traduzem os valores da PA desta população.

Já a disparidade de resultados de 1975 e 1976 relativos aos individuos de cor parda ou amarela não permite qualquer conclusão quanto à influência da idade e sexo no comportamento da PA nestes dois grupos (Tabela 5).

Vários autores têm assinalado a diticuldade de definir a influência da cor negra e nesta cor a influência do sexo e idade sobre os niveis da PA e prevalência da hipertensão, quando são comparados os resultados de pesquisas levadas a efeito em locais com características epidemiológicas e sócio-econômicas diferentes $5,13,18$. Levandose em conta que, entre 1975 e 1976, não ocorreram em Botucatu surtos migratórios, de transformação econômica ou social ou outros fatores que pudessem ter influido nas características epidemiológicas da população, as disparidades dos resultados de 1975 e 1976 devem ser devidas à exiguidade da parcela negra e amarela da população. Verifica-se desta forma a influência do tamanho da amostra na exatidão dos resul- tados obtidos. Estes resultados reafirmam a necessidade da realização de estudos semelhantes em outras regiões do país, onde os contingentes de negros, pardos ou amarelos sejam mais expressivos.

A influência do tamanho da amostra evidencia-se também nas diferenças de níveis da PA obtidas em 1975 e 1976, para os obesos de cor branca e para todos os pesos da cor parda. Apesar destas disparidades, a tendência de num mesmo sexo as pressões serem maiores quanto maior o peso foi observada em ambos os anos tanto na parcela branca quanto na negra da população, confirmando as conclusões quanto à influência do peso a que se chegou anteriormente ${ }^{3}$.

A prevalência de hipertensão e de limítrofes em 1976 foi superior a de 1975. Este pequeno aumento de prevalência não pode ser explicado por diferenças na composição dos grupos estudados quanto a sexo e idade. Quanto à cor, em 1976, a parcela negra da população em estudo passou de $2,7 \%$ a $5,2 \%$ sofrendo un aumento de $92,5 \%$; no entanto, devido à exiguidade do contingente de cor negra a diferença nãu pode ser atribuída a este acréscimo verjficado.

Al.meIDA, D. B. de et al. [Blood pressure levels and hipertension prevalence in 16 to 25 year-old students in Botucatu, S. Paulo, Brazil. A comparative study of two successive cohorts, 1975-1976.J Rev. Saúde públ., S. Patulo, 12: 497-505, 1978 .

ABSTRACT: To determine the prevalence of hypertension and blood pressure levels in a young population group, two groups, one of 1288 students and another of 736 , all from Botucatu, were investigated during two consecutive years, and the results were then compared. The mean values for systolic readings of the whole population, as for the two age groups (16 to 20 and 21 to 25 years old), were identical for both years studied; the diastolic mean values differed by 2 $\mathrm{mmHg}$ at most. The mean systolic and diastolic reading for both sexes and among the whites also differed by $2 \mathrm{mmHg}$ at most. The mean values for the whole population and among the white population were higher for males than for females, and also higher in the 21 to 25 age group than in the 16 to 20 group. The 1975 and 1976 results for the black and yellow population were inconsistent, reflecting the influence of the small size of these groups. The prevalence of arterial hypertension (systolic readings equal to or higher than $140 \mathrm{mmHg}$ and diastolic readings equal to or higher than $90 \mathrm{mmHg}$ ) was $5.04 \%$ in 1975 and $6.22 \%$ in 1976, being higher in the males than in the females and higher in the 21 to 25 age group than in the 16 to 20 year-old group.

UNITERMS: Hypertension. Blood pressitre. Students, Botucatu, SP, Brazil. 
ALmeidA, D. B. de et al. Estudo comparativo da pressão arterial e da prevalência de hipertensão arterial em duas coortes sucessivas (1975-1976) de estudantes de 16 a 25 anos, Botucatu, SP, Brasil. Rev. Saúde puibl., S. Paulo, 12:49'\%-505, 1978.

\section{REFERENCIAS BIBLIOGRÁFICAS}

1. ACOSTA, M. I. T. Diagnóstico de saúde em membros de um grupo de familias do bairro "Jardim das oliveiras", Municipio de Campinas, Estado de São Paulo: comentarios sobre aspectos metodológicos e técnicos concernentes. Campinas, 1972. [Tese de Doutoramento - Faculdade de Ciências Médicas UNICAMP].

2 AGNE, L. R. et al, Censo tensiométrico em funcionários da indústria da "Grande Porto Alegre". In: Congresso Brasileiro de Nefrologia, $7^{\circ}$, Curitiba, 1976. Resumos. Curitiba, 1976. p. 40.

3. ALMEIDA, D. B. de et al. Niveis pressóricos de estudantes de 16 a 25 anos da área urbana do município de Botucatu, São Paulo, Brasil, em 1975. [Aceito para publicação no Boletim de la Oficina Sanitaria Panamericana].

4. CARDMAN, R. et al. Hipertensão arterial e outros fatores de risco coronáriano em 10.000 pacientes. Arq. bras. Cardiol., 30 (Supl. 2):363, 1977.

5. CHAKRABORTY, $R$, et al. Heredity stress and blood pressure; a family set method. V-Heritability estimates. $J$. cron. Dis., 30:683-99, 1977.

6. CARVAlHo, J. J. M. et al. Influência de fatores ambientais na hipertensão arterial. Arq. bras. Cardiol., 30(Supl. 2) : 347,1977

7. CAVALCANTE, W. J, et al, Estudo epidemiológico da pressão arterial em crianças, Arq. bras. Cardiol., 30 (Supl. 2) : 346,1977

8. COLLI, A. S. et al. Freqüuencia cardíaca e pressão arterial de adolescentes. Arq. bras, Cardiol., 28:37-44, 1975.

9. CORTEZ JR., L. S. Mortalidade, segundo causas múltiplas, no Municipio de Botucatu, SP. Campinas, 1976. [Tese de Doutoramento - Faculdade de Ciências Médicas da UNICAMP]

10. $\operatorname{COSTA}$, C. Hipertensão arterial sistêmica. Prevalência nas unidades de internação do Hospital das Clínicas de Porto Alegre. [Apresentado no I Simpósio sobre Hipertensão Arterial, Salvador. Bahia, 1977].
11. GONZALEZ, M. A pressão arterial nos escolares de Porto Alegre. Rev. Med. Rio Grande do Sul, p. 178-80, mar. ahr. 1948

12. $\mathrm{HADDAD}, \mathrm{N}$. Inquérito epidemiológico sobre cardiopatias crônicas em um bairro de Ribeirão Preto, SP, Brasil. Arq. Hig., S. Paulo, 32/33:27-77, $1967 / 8$.

13. HARBURG, E. et al. A family set method for estimating heredity and stress. I-A pilot survey of blood pressure among negroes in high and low stress areas, Detroit 1966-1967. J. chron. Dis., 23:69-81, 1970.

14. MACHADO, D. F. Prevalência de hipertensão arterial sistêmica em mulheres grávidas da maternidade Climério de Oliveira e do Hospital Ana Nery de Salvador (Bahia), GO, 4:9-58, 1970.

15. MAGALDI, C. \& ALMEIDA, D. B. Aspecpectos epidemiológicos e preventivos da doença hipertensiva. [Enviado para publicação nos Arquivos Brasileiros de Cardiologia].

16. A MEDIDA da pressão arterial nas populações paulistana e carioca. Arq. bras. Cardiol., 28:399-402, 1975.

17. NOGUEIRA, J. L. Levantamento epidemiológico sobre cardiopatias e pressão arterial na populacão do municipio de Cassia dos Coqueiros, SP, Brasil. Ribeirão Preto, 1972. [Tese de Doutoramento -- Faculdade de Medicina de Ribeirão Preto USP]

18. REICHMAN, L. B. et al. Hypertension testing among high school students. I - Surveillance procedures and results. J. chron. Dis., 28:161-71, 1975.

19. SILVA, G. R. Indices de morbilidade em um grupo de famílias na oidade de Salvador. Salvador, 1961. [Tese livredocência - Faculdade de Medicina Univ. Fed. Bahia].

Recebido para publicąão em 23/02/1978

Aprovado nara publicacão em 09/08/1978 